\title{
Adjuvant Chemotherapy Using the FOLFOX Regimen in Colon Cancer
}

\author{
Hyeong-Joon Jeon, Jin-Hee Woo, Hak-Yoon Lee, Ki-Jae Park, Hong-Jo Choi \\ Department of Surgery, Dong-A University Medical Center, Busan, Korea
}

Purpose: Great progress has been made in the adjuvant treatment of colon cancer. The aim of this study was to evaluate the efficacy of postoperative adjuvant chemotherapy using the FOLFOX regimen in patients with stage III and high-risk stage II colon cancer.

Methods: Eighty-two patients who underwent a potentially curative resection for stage III or high-risk stage II colon cancer were enrolled in this retrospective study. They received FOLFOX4 or modified FOLFOX6. The primary endpoint was disease-free survival.

Results: During the median follow-up of 37 months (range, 21 to 61 months), 14 patients experienced disease relapse. The disease-free survival rate at 3 years was $82.9 \%: 84.6 \%$ for stage II and $82.6 \%$ for stage III. At the time of the analysis, 8 patients were dead from recurrence. The probability of overall survival at 5 years was $74.5 \%: 90 \%$ for stage II and $74.6 \%$ for stage III. Grade 3 or 4 hematologic adverse events included neutropenia (40.2\%), anemia (2.4\%), and thrombocytopenia (1.2\%). Gastrointestinal toxicities included grade 3 or 4 nausea (4.9\%) and stomatitis (2.4\%). Peripheral sensory neuropathy was observed in $81.7 \%$ of the patients during treatment. Of the 11 patients $(13.4 \%)$ who had grade 3 peripheral sensory neuropathy during treatment, grade 3 symptoms were persistent in 3 patients with gait disturbance at the time of analysis. No treatment-related deaths were recorded.

Conclusion: Postoperative chemotherapy using the FOLFOX regimen, oxaliplatin in combination with 5-fluorouracil and leucovorin, is effective and tolerable in patients with stage III and high-risk stage II colon cancer.

\section{Keywords: Colonic neoplasms; Adjuvant chemotherapy; Oxaliplatin; FOLFOX protocol}

\section{INTRODUCTION}

Colorectal cancer (CRC) is the third most common malignancy in Korea and accounted for approximately 22,000 new cancer cases and 6,800 deaths in 2008 [1]. As many as $40 \%$ to $50 \%$ of patients who undergo a potentially curative surgery alone will ultimately relapse and die of metastatic disease [2].

Over the past years, considerable progress has been made in the adjuvant treatment of patients with colon cancer. Following the

Received: May 27, 2011 Accepted: June 13, 2011

Correspondence to: Hong-Jo Choi, M.D.

Department of Surgery, Dong-A University Medical Center, 1

Dongdaesin-dong 3-ga, Seo-gu, Busan 602-715, Korea

Tel: +82-51-240-5146, Fax: +82-51-247-9316

E-mail: colonch@donga.ac.kr

(c) 2011 The Korean Society of Coloproctology

This is an open-access article distributed under the terms of the Creative Commons Attribution NonCommercial License (http://creativecommons.org/licenses/by-nc/3.0) which permits unrestricted non-

commercial use, distribution, and reproduction in any medium, provided the original work is properly cited.
Intergroup trial (INT-0035) for adjuvant 5-fluorouracil (5-FU) plus levamisole therapy in patients with stage III colon cancer [3], 5-FU plus leucovorin (LV) combination (FL) therapy became the standard chemotherapy for stage III colon cancer $[4,5]$. Beginning with new millennium, oxaliplatin, a third-generation platinum derivative alkylating agent that inhibits DNA replication, emerged as one of the most effective chemotherapeutics (in terms of response rate and progression-free survival) for metastatic colorectal cancer when combined with 5-FU and LV $[6,7]$. To determine if oxaliplatin in combination with 5-FU and LV can also show improved efficacy in adjuvant settings, Andre et al. [8] conducted an international phase III clinical trial, the MOSAIC (the Multicenter International Study of Oxaliplatin/5-Fluorouracil/Leucovorin in the Adjuvant Treatment of Colon Cancer) study and demonstrated that adding oxaliplatin to a regimen of bolus and continuous-infusion 5-FU combined with LV (FOLFOX4) improved disease-free survival (DFS) significantly in stage II and III colon cancer compared with the same regimen of 5-FU and LV (LV5FU2). Based on the results of the MOSAIC study, the US Food and Drug Administration ap- 
proved the FOLFOX4 regimen for postoperative adjuvant therapy in patients with stage III colon cancer in November 2004. Additional follow-up of the MOSAIC trial also proved the persistent DFS benefit of the FOLFOX4 regimen in the adjuvant treatment of colon cancer and confirmed that this effect translated into an overall survival (OS) benefit [9]. The aim of this retrospective study was to evaluate the efficacy and the toxicity of the FOLFOX regimen as postoperative adjuvant chemotherapy in patients with stage III and high-risk stage II colon cancer on whom a potentially curative surgical resection had been performed.

\section{METHODS}

Patients were eligible when they had undergone a potentially curative surgical resection of pathologically proven stage III (any TN1/ $2 \mathrm{M} 0$ ) or high-risk stage II colon cancer. Stage II disease was classified as high risk when it had at least one of the following factors: $\mathrm{T} 4 \mathrm{a} / 4 \mathrm{~b}$, tumor perforation, bowel obstruction, a poorly-differentiated tumor, and venous or lymphatic invasion. As in the MOSAIC trial [8], colon cancer was defined by the presence of the inferior margin of the tumor above the peritoneal reflection. Other eligibility criteria included age between 18 and 79 years, Eastern Cooperate Oncology Group performance scale of two or less, the absence of prior chemotherapy, immunotherapy or radiotherapy, and adequate blood counts and liver and kidney functions. Before treatment, written informed consent, based on information about chemotherapeutic drugs, treatment schedule and toxicity, was provided by all patients. For safe delivery of the chemotherapeutic drugs, a central venous port was implanted into the subclavian vein in all patients. Treatment was started not later than eight weeks after surgery.

\section{Treatment}

Patients received the FOLFOX4 or the modified FOLFOX6 (mFOLFOX6) regimen. Both original oxaliplatin (Eloxatin ${ }^{\circledR}$, SanofiAventis, Paris, France) and generic oxaliplatin $\left(\mathrm{OXP}^{\circledR}\right.$, Shinpoong Pharmaceutical Co., Seoul, Korea) were administered randomly. The FOLFOX4 regimen consisted of LV, $200 \mathrm{mg} / \mathrm{m}^{2} /$ day given as a 2-hour infusion, followed by a bolus $5-\mathrm{FU}, 400 \mathrm{mg} / \mathrm{m}^{2}$, and a 22 hour continuous infusion of 5-FU, $600 \mathrm{mg} / \mathrm{m}^{2}$, repeated for 2 consecutive days. Oxaliplatin, $85 \mathrm{mg} / \mathrm{m}^{2}$, was administered on day 1 only and was given as a 2-hour infusion in $250 \mathrm{~mL}$ of dextrose $5 \%$, concurrent with LV. The mFOLFOX6 regimen included oxaliplatin, $85 \mathrm{mg} / \mathrm{m}^{2}$ in $250 \mathrm{~mL}$ of dextrose $5 \%$, concurrent with LV, 400 $\mathrm{mg} / \mathrm{m}^{2}$, given as a 2 -hour intravenous infusion on day 1 , followed by a bolus $5-\mathrm{FU}, 400 \mathrm{mg} / \mathrm{m}^{2}$, on day 1 and a continuous $5-\mathrm{FU}, 1,200$ $\mathrm{mg} / \mathrm{m}^{2} /$ day, on day 1 and 2 (46-hour continuous infusion of a total 5-FU of 2,400 mg/m²). Oxaliplatin and LV were given separately via a Y-connector. In each cycle, routine antiemetic prophylaxis was performed with a 5-hydroxytryptamine 3 receptor antagonist (granisetron hydrochloride). The cycles were repeated every 2 weeks.
Before starting each cycle, patients were assessed by using the $\mathrm{Na}$ tional Cancer Institute common toxicity criteria. Dose modifications were performed according to predefined guidelines based on toxicities, as described in the MOSAIC trial [8]. Dose reductions were based on the worst adverse effects observed during the previous cycle. The dose of oxaliplatin was reduced by $20 \%$ in cases of persistent (at least 14 days) paresthesia, temporary painful paresthesia or functional impairment. Together with reductions in the dose of oxaliplatin, both the bolus and the infusion dose of 5FU were reduced by $20 \%$ in the event of grade $3 / 4$ neutropenia or thrombocytopenia (or both), diarrhea, stomatitis, or other drugrelated adverse effects of grade 3 . Chemotherapy was delayed until recovery if neutrophils decreased to less than $1.5 \times 10^{9} / \mathrm{L}$ or platelets decreased to less than $100 \times 10^{9} / \mathrm{L}$ or for significant persisting nonhematologic toxicity. In cases of persistent painful paresthesia or functional impairment even after dose reduction of the oxaliplatin, the regimen was switched to the Mayo Clinic regimen or capecitabine monotherapy.

\section{Follow-up}

Patients were assessed before adjuvant treatment, every two weeks during treatment, and then every three months for three years. During treatment, medical history, physical examination, neurologic assessment, $\mathrm{CBC}$, and liver and kidney function tests were assessed at baseline and were repeated every cycle, and the serum carcinoembryonic antigen (CEA) level and abdominopelvic, and low-dose chest computed tomography (CT) were assessed every six cycles. After completion of the chemotherapy, specific oncologic assessment included serum CEA level and chest radiography every three months, and abdominopelvic and low-dose chest CT biannually. Patients had colonoscopy postoperatively at one year, and thereafter as indicated. Patients were monitored for adverse effects throughout the treatment period and until 28 days after the last cycle of chemotherapy unless treatment-related adverse effects required additional follow-up. The diagnosis of recurrence was made on the basis of imaging studies and, if indicated, biopsy.

\section{Statistical analysis}

Data were entered into a spreadsheet program and subsequently imported into a statistic programs (Prism ${ }^{\mathrm{TM}}$, version 4; GraphPad Software, San Diego, CA, USA). The primary end-point was DFS, defined as the time from treatment to relapse of colon cancer or death as a result of any cause. Secondary efficacy variables were safety, including long-term adverse effects, and overall survival. Safety analyses included patients who had received at least one cycle of treatment. Survivals were calculated using the Kaplan-Meier method and were analyzed using the log-rank test.

\section{RESULTS}

\section{Study population}

Eighty-two patients were enrolled in this retrospective study from 
April 2006 to December 2008. The baseline characteristics of the enrolled patients are shown in Table 1. The median age was 59 years (range, 28 to 79 years), and the most common site of the primary tumor was the sigmoid colon ( 42 patients), followed by the rightsided colon (16 patients) and the rectosigmoid junction (12 patients). A laparoscopic colectomy was performed in 49 patients (conversion was necessary in 3 patients) and an open colectomy in 33.

Postoperative pathologic characteristics are detailed in Table 2. Sixty-nine patients were confirmed to have stage III disease and 13 to have high-risk stage II disease. High-risk factors in stage II included lymphatic/vascular invasion in eight patients, T4b tumors in four patients, and obstruction and perforation in two patients.

The details of FOLFOX chemotherapy are presented in Table 3. As to the regimen, the FOLFOX4 regimen was administered in 56 patients and the mFOLFOX6 regimen in 26. Patients received at least one cycle of the FOLFOX regimen, and 67 patients (82\%) were able to receive at least 10 of 12 cycles of the FOLFOX regimen, with a median of 12 cycles (range, 1 to 12 cycles). For oxaliplatin preparation, Eloxatin ${ }^{\circledR}$ was administered in half of the patients and $\mathrm{OXP}^{\circledR}$ in the remaining half.

\section{Toxicity}

As shown in Table 4, the most frequent adverse events during the

Table 1. Baseline characteristics of patients

\begin{tabular}{lc}
\hline Variable & No. (\%) \\
\hline Median age (range, yr) & $59(28-79)$ \\
Age (yr) & \\
$<65$ & $54(65.9)$ \\
$\geq 65$ & $28(34.1)$ \\
Sex & \\
Male & $48(58.5)$ \\
Female & $34(41.5)$ \\
Anatomic tumor location & \\
\hline Right colon & $16(19.5)$ \\
Transverse colon & $3(3.7)$ \\
Left colon & $9(11)$ \\
Sigmoid colon & $42(51.2)$ \\
Rectosigmoid junction & $12(14.6)$ \\
Preoperative serum CEA (ng/mL) & \\
$<5$ & $68(82.9)$ \\
$\geq 5$ & $14(17.1)$ \\
Method of surgery & \\
\hline Laparoscopic colectomy a & $49(59.8)$ \\
Open colectomy & $33(40.2)$ \\
\hline
\end{tabular}

CEA, carcinoembryonic antigen.

${ }^{a}$ Three cases of conversion were included. treatment were peripheral sensory neuropathy (PSN; $81.7 \%)$, neutropenia $(74.4 \%)$, diarrhea (65.9\%), and nausea (62.2\%). There were no differences in toxicity profiles between patients who received the FOLFOX4 regimen and those who received the mFOLFOX6 regimen. Grade 3/4 hematologic adverse events included neutropenia $(40.2 \%)$, anemia $(2.4 \%)$, and thrombocytopenia (1.2\%). Gastrointestinal toxicities included grade $3 / 4$ nausea (4.9\%), stomatitis (2.4\%). Of the 11 patients (13.4\%) who had grade 3 PSN during treatment, grade 3 symptoms were still present in 3 patients with gait disturbance at the time of analysis. No treatment-related deaths were recorded.

Embarrassingly, a catheter embolization to the pulmonary artery developed in a 37-year-old male patient with stage IIC disease. Retrospective radiologic review of plain chest radiographs confirmed evidence of compression of the catheter at the costo-clavicular junction (pinch-off syndrome) (Fig. 1A). He did not complain of any clinical manifestations associated with it, and the embolized fragment was found incidentally by routine chest radiography several

Table 2. Pathologic characteristics of patients

\begin{tabular}{lc}
\hline Variable & No. \\
\hline Histologic differentiation & 27 \\
Well differentiated & 45 \\
Moderately differentiated & 3 \\
Poorly differentiated & $6 / 1$ \\
Mucinous/signet ring cell & \\
Depth of invasion & 4 \\
T1 & 4 \\
T2 & 61 \\
T3 & $13(8 / 5)$ \\
T4 (T4a/T4b)
\end{tabular}

No. of nodes involved

\begin{tabular}{lc}
0 & 13 \\
$1-4$ (N1a/N1b) & $46(28 / 18)$ \\
$\geq 5$ (N2a/N2b) & $23(13 / 10)$ \\
Lymphatic invasion (-/+) & $48 / 34$ \\
Vascular invasion (-/+) & $65 / 17$ \\
Perineural invasion (-/+) & $65 / 17$ \\
p53 mutation (-/+) & $28 / 54$ \\
Disease stage & \\
Stage II (IIA/IIC) & $13(9 / 4)$ \\
Stage III (IIIA/III/IIIC) & $69(6 / 50 / 13)$ \\
High risk factor in stage II & \\
Obstruction & 2 \\
Perforation & 2 \\
Lymphatic/vascular invasion & 8 \\
T4b & 4 \\
\hline
\end{tabular}


months after the completion of 12 cycles of the FOLFOX4 regimen (Fig. 1B). The embolized catheter was successfully retrieved by using an interventional radiologic approach (Fig. 2).

\section{Outcome}

Fourteen patients experienced disease relapse during the median follow-up of 37 months (range, 21 to 61 months). The diverse patterns of relapse are shown in Table 5. The Kaplan-Meier curve for 82 patients is shown in Fig. 3A. DFS rates at 2 and 3 years were $84.1 \%$ and $82.9 \%$, respectively. No significant difference in DFS was observed between patients who received the FOLFOX4 regimen and those who received the mFOLFOX6 regimen. The 3-year DFS rates were $83.9 \%$ for the FOLFOX 4 regimen and $80.8 \%$ for the mFOLFOX6 regimen ( $\mathrm{P}=0.755)$. The Kaplan-Meier estimates of the DFS rates at 3 years according to stage are shown in Fig. 3B: $84.6 \%$ for stage II and $82.6 \%$ for stage III (hazard ratio [HR], 0.873 ; $\mathrm{P}=0.86$ ). At the time of analysis, eight patients had died due to recurrence, but no patients had died without recurrence. The probabilities of OS at 3 and 5 years were $87.5 \%$ and $74.5 \%$, respectively. According to stage, estimates of OS at 5 years were $90 \%$ for stage II and $74.6 \%$ for stage III.

Table 3. Details of the FOLFOX chemotherapy

\begin{tabular}{ll}
\hline Variable & No. (\%) \\
\hline FOLFOX regimen & \\
FOLFOX4 & $56(68.3)$ \\
Modified FOLFOX6 & $26(31.7)$ \\
Median cycles of chemotherapy (range) & $12(1-12)$ \\
Product of oxaliplatin & \\
Original (Eloxatin $\left.{ }^{\circledR}\right)$ & $41(50)$ \\
Generic $\left(\right.$ OXP $\left.^{\circledR}\right)$ & $41(50)$ \\
\hline
\end{tabular}

\section{DISCUSSION}

Adjuvant chemotherapy given after a potentially curative surgery was developed to reduce the risk of disease relapse, either as local recurrences or distant metastases, in patients with stage II and III colon cancer. A traditional endpoint of adjuvant clinical trials for colon cancer has been 5-year OS. Recent adjuvant trials, however, use 3-year DFS as the primary endpoint on the grounds that relapse of disease occurs within the first 3 years in the majority of patients, with the relapse rate reaching a peak in the second year [10], and that a recent analysis of pooled data by Sargent et al. [11] from over 20,000 patients with stage III colon cancer found that 3-year DFS highly correlated with 5-year OS, suggesting that 3-year DFS is an appropriate primary endpoint for adjuvant colon cancer clinical trials of fluoropyrimidine-based regimens. Most recently,

Table 4. Adverse events

\begin{tabular}{lcc}
\hline Adverse events & All grades & Grades 3 and 4 \\
\hline Hematologic & $61(74.4)$ & $33(40.2)$ \\
Neutropenia & $38(46.3)$ & $2(2.4)$ \\
Anemia & $24(29.3)$ & $1(1.2)$ \\
Thrombocytopenia & & \\
Gastrointestinal & $54(65.9)$ & $1(1.2)$ \\
Diarrhea & $51(62.2)$ & $4(4.9)$ \\
Nausea & $12(14.6)$ & $1(1.2)$ \\
Vomiting & $30(36.6)$ & $2(2.4)$ \\
Stomatitis & $67(81.7)$ & $11(13.4)$ \\
Sensory neuropathy & $4(4.9)$ & $\mathrm{NA}$ \\
Alopecia & & \\
\hline
\end{tabular}

Values are presented as number (\%). NA, not applicable.
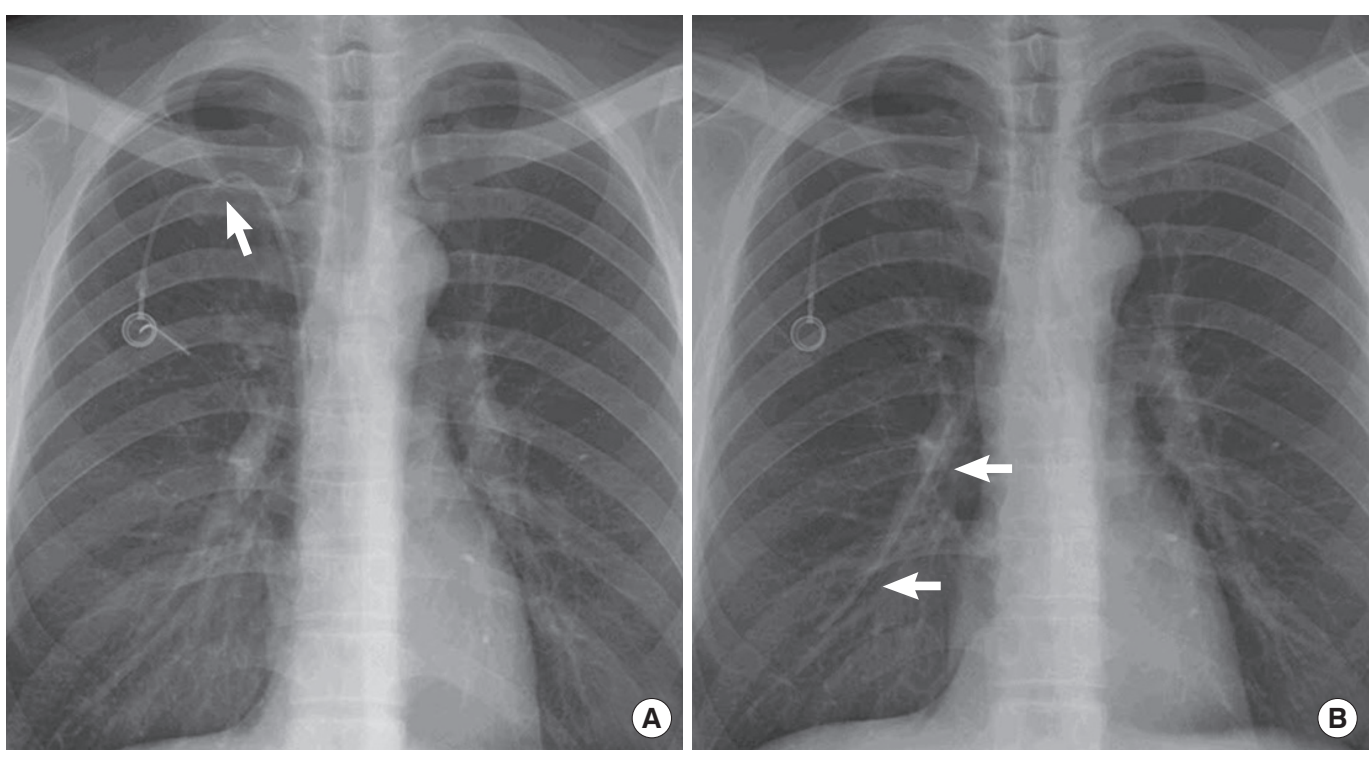

Fig. 1. Catheter fracture due to pinch-off syndrome. (A) The catheter was entrapped at the point where the catheter crosses between the clavicle and the first rib (arrow). (B) Distal portion of the catheter was fractured and embolized to the right pulmonary artery (arrows). 

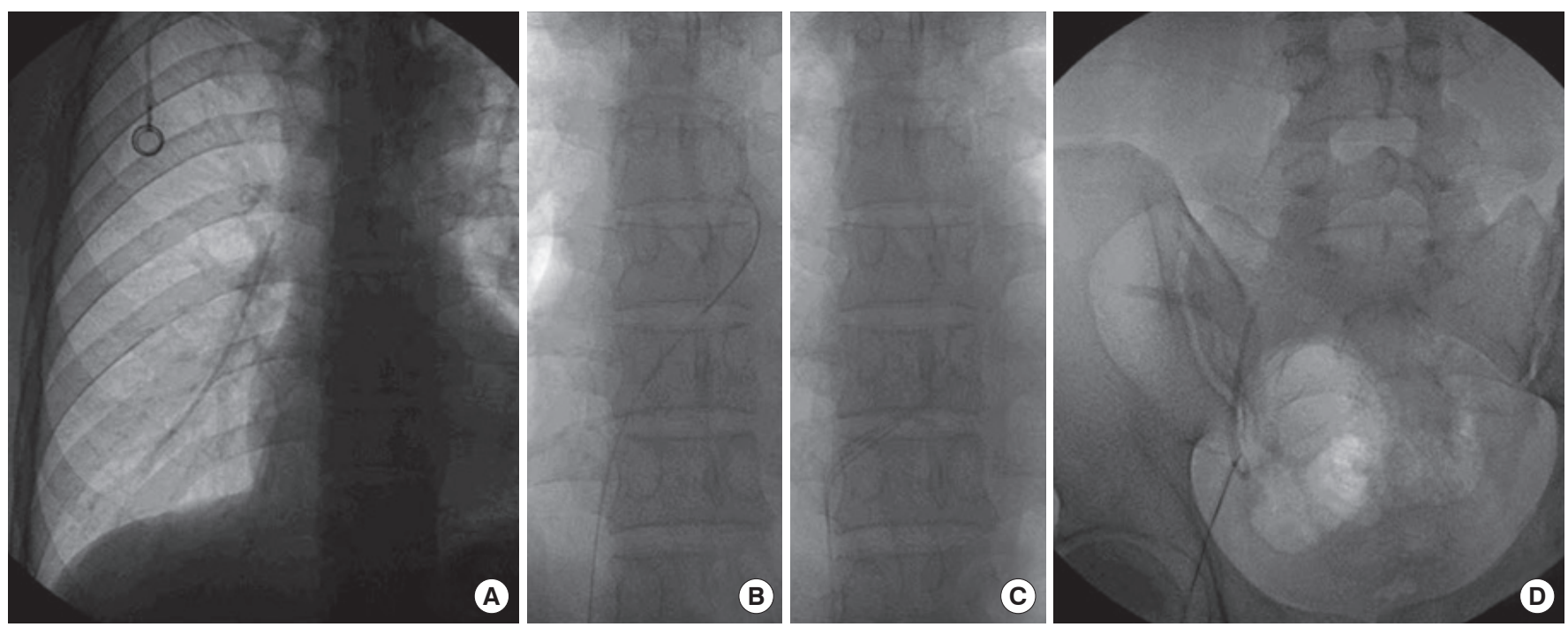

Fig. 2. Port catheter retrieval by using an interventional radiologic approach. (A) A direct radiograph shows the dislodged port catheter within the right pulmonary artery. (B-D) By catching the displaced tip with a gooseneck snare, we retrieved the catheter through the right femoral vein.
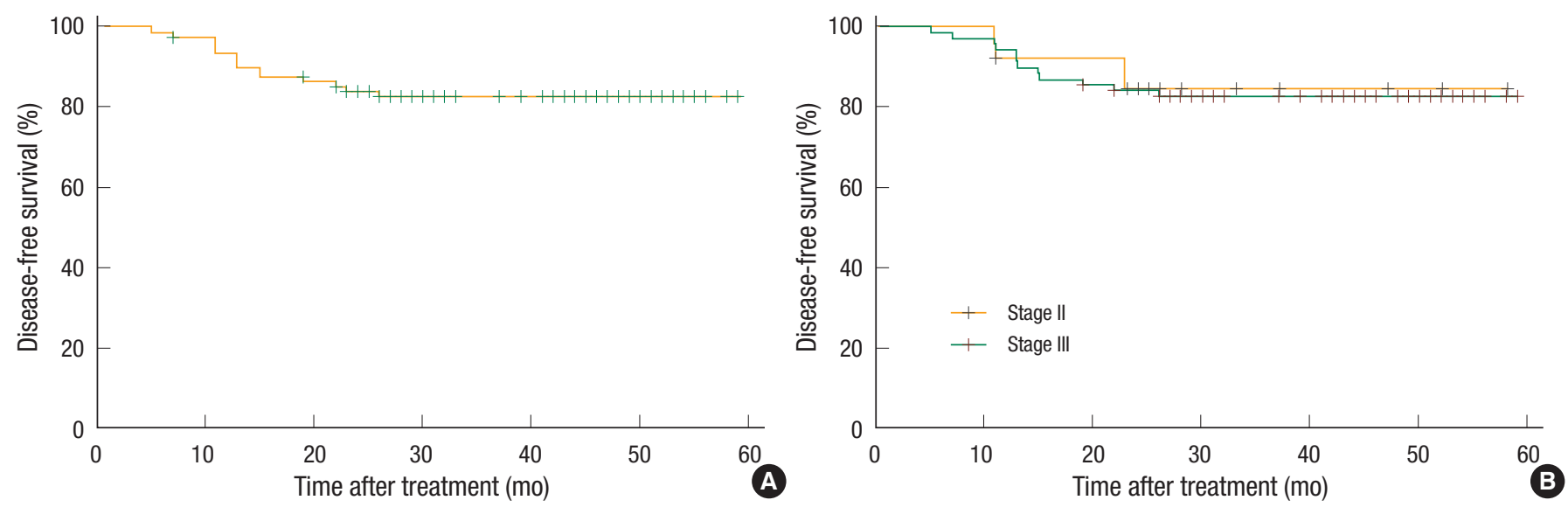

Fig. 3. Kaplan-Meier curve for disease-free survival (A) in all patients and (B) by stage.

Table 5. Patterns of relapse

\begin{tabular}{ll}
\hline Lung metastases & 3 \\
Systemic lymph nodes metastases & 3 \\
Liver metastases & 2 \\
Liver metastases + peritoneal seeding & 1 \\
Multiple bone metastases + peritoneal seeding & 1 \\
Multiple bone metastases & 1 \\
Peritoneal seeding & 1 \\
Krukenberg tumor & 1 \\
Regional recurrence & 1 \\
\hline
\end{tabular}

Sargent et al. [12], from six trials of 12,676 patients, validated that results based on DFS assessed after 2 or 3-year median follow-up are appropriate primary endpoints for adjuvant stage III colon cancer trials with 5-FU with or without oxaliplatin or irinotecan.

The MOSAIC trial demonstrated the superiority of oxaliplatin in FOLFOX4, compared to LV5FU2, in improving DFS in patients with resected stage II and III colon cancer [8]. The DFS at 3 years was $78.2 \%$ for the FOLFOX regimen versus $72.9 \%$ for the LV5FU2 regimen. The FOLFOX4 regimen increased the probability that a patient would be alive and disease-free at 3 years by $5.3 \%$. The HR for recurrence in the group given the FOLFOX regimen, as compared with the LV5FU2 group, was 0.77 (95\% confidence interval [CI], 0.65 to $0.91 ; \mathrm{P}=0.002)$; treatment with the FOLFOX regimen resulted in a $23 \%$ reduction in the relative hazard of a DFS event. The probability of OS at 3 years was $87.7 \%$ for the FOLFOX regimen and $86.6 \%$ for the LV5FU2 regimen.

The National Surgical Adjuvant Breast and Bowel Project 07 (NSABP-07) trial comparing the oxaliplatin and bolus 5-FU/LV 
(FLOX) regimen to the bolus 5-FU/LV (FULV, Roswell Park regimen) regimen for resected stage II and III colon cancer also demonstrated the superiority of an oxaliplatin-based regimen in the adjuvant treatment of colon cancer [13]. Three-year DFS was 76.1\% in the FLOX group compared to $71.8 \%$ in the FULV arm; compared to the FULV regimen, the FLOX regimen increased the probability that a patient would be alive and disease free at 3 years by $4.3 \%$. The relative hazard of the DFS event was reduced by $20 \%$ with FLOX treatment as compared to FULV treatment, with a HR of 0.80 ( $95 \% \mathrm{CI}, 0.69$ to $0.93 ; \mathrm{P}=0.0034$ ). These data confirm that a significant DFS benefit can be attained when oxaliplatin is added to fluoropyrimidine-based regimens.

The DFS and the OS rates at 3 years in the present retrospective study were $82.9 \%$ and $87.5 \%$, respectively, and these results are favorably compared with those of the MOSAIC and the NSABP-07 trials. The updated results of the MOSAIC trial showed a trend toward improvement in survival for patients treated with the FOLFOX regimen [9]. The DFS rates at 5 years were $73.3 \%$ for the FOLFOX regimen and $67.4 \%$ for the LV5FU2 regimen ( $\mathrm{HR}, 0.80$; $95 \% \mathrm{CI}$, 0.68 to $0.93 ; \mathrm{P}=0.003)$. The $\mathrm{OS}$ rates at 6 years were $78.5 \%$ for the FOLFOX regimen and $76.0 \%$ for the LV5FU2 regimen (HR, 0.84; $95 \% \mathrm{CI}, 0.71$ to $1.00 ; \mathrm{P}=0.046$ ). Similar results were obtained in the current study and the probability of OS at 5 years was $74.5 \%$.

In the MOSAIC trial, the significant DFS and OS benefits in the overall FOLFOX group were driven entirely by the favorable effect in the subgroup of patients with stage III disease. The clinical benefit of the FOLFOX4 regimen, compared with the LV5FU2 regimen, in terms of 6-year OS and 5-year DFS reached statistical significance and clinical relevance only among stage III patients. For stage II patients, there were no statistically significant improved 5 -year DFS and 6-year OS. The role of postoperative adjuvant therapy for stage II colon cancer still remains controversial, and the MOSAIC trial showed only a trend toward improved DFS at 5 years in patients with high-risk stage II disease treated with the FOLFOX4 regimen, as compared to patients treated with the LV5FU2 regimen. As suggested by Andre et al. [9], future studies to identify high-risk stage II disease patients to evaluate oxaliplatinbased adjuvant chemotherapy in this subgroup of patients are necessary.

Diverse spectra of toxicities were observed in chemotherapy with oxaliplatin in combination with 5-FU and LV. The toxicity profile of the FOLFOX4 regimen seen in the MOSAIC trial is somewhat distinct from that seen with the FLOX regimen in the NSABP-07 trial. One of the most specific safety concerns associated with oxaliplatin-based chemotherapy is PSN. PSN was observed in 92\% of the patients who received the FOLFOX regimen and over 12\% was grade 3 , compared with $15.6 \%$ with $0.2 \%$ grade 3 for the LV5FU2 arm [8]. Fortunately, this toxicity showed improvement with time. At one year, 11 patients (1.1\%) continued to have grade 3 peripheral neurosensory symptoms, and this declined to 7 patients $(0.7 \%)$ at 18 months [9]. In the NSABP-07 trial, grade 3 PSN was seen in $8.2 \%$ of patients and grade 4 in $0.2 \%$ of patients receiving the FLOX regimen, compared with $0.7 \%$ with grade 3 PSN only in the FULV regimen [13]. At 1 year from random assignment, the rate of grade 3 PSN in the FLOX arm was $0.6 \%$. The lower incidence of PSN seen in the NSABP C-07 trial was a likely result of the lower cumulative dose of oxaliplatin given in the NSABP C-07 trial (nine planned doses of $85 \mathrm{mg} / \mathrm{m}^{2}$ ) than in the MOSAIC trial (12 planned doses of $85 \mathrm{mg} / \mathrm{m}^{2}$ ) [13]. Conversely, more grade $3 / 4$ diarrhea was observed with the FLOX regimen in the NSABP C-07 trial (38\%) than was observed with the FOLFOX regimen in the MOSAIC trial (10.8\%) and might be the result of different treatment schedules. Furthermore, the FLOX regimen was associated with an increased risk of bowel wall injury (BWI) syndrome, which has not been reported for the FOLFOX4 regimen, and five among 15 deaths in the FLOX arm were attributed to chemotherapy-induced enteropathy [13].

Although PSN developed in $81.7 \%$ of patients during treatment in the current study, $83.5 \%$ of them were of grade $1 / 2$. However, 3 of the 11 patients with grade 3 PSN were still complaining of gait disturbance associated with PSN at the analysis. In the MOSAIC trial, grade $3 / 4$ neutropenia was observed in $41 \%$ of the patients in the FOLFOX arm compared with less than $5 \%$ of the patients in the LV5FU2 arm; neutropenic infection or fever was, however, rare in both groups ( $1.8 \%$ and $0.2 \%$, respectively) [8]. In the NSABP-07 trial, infection associated with grade $3 / 4$ neutropenia was seen in $1.0 \%$ of patients receiving the FULV regimen and in $2.2 \%$ of patients receiving the FLOX regimen [13]. Similarly, grade 3/4 neutropenia was seen in $40.2 \%$ in the present study, but no neutropenic infection or fever developed. The all-cause mortality during treatment with each of the oxaliplatin-containing regimens was low and was the same as in the control arms $(0.5 \%$ for the FOLFOX4 regimen in the MOSAIC trial and 1.2\% for the FLOX regimen in the NSABP C-07 trial) $[8,13]$. No treatment-related deaths were recorded in this study.

In addition to toxicities associated with chemotherapeutic agents, complications associated with central venous port systems need to be taken into consideration. We experienced a case of catheter fracture and embolization of the distal fragment to the right pulmonary artery. Catheter fracture can be caused by entrapment of the catheter between the first rib and the clavicle (pinch-off syndrome). Biffi et al. [14] reported this complication in 9 out of 1,320 subclavian port placements $(0.68 \%)$. Usually, patients have no symptoms from the embolized catheter fragment itself, but when catheter embolization develops during treatment, it may have disastrous results; leakage of chemotherapeutic drugs out into the surrounding tissue can cause extensive necrosis [15]. In our patient, fortunately, catheter embolization developed several months after the completion of scheduled chemotherapy, and the embolized catheter was successfully retrieved by using an interventional radiologic approach. The radiologic retrieval of embolized fragments is the preferred method of treatment, and it has a high success rate [14]. Here, we can take a lesson in the care of the central venous port system; it should be removed as soon as possible after sched- 
uled chemotherapy has been completed.

The oncologic outcomes of oxaliplatin, in combination with 5$\mathrm{FU} / \mathrm{LV}$, in this retrospective study were consistent with those in several trials, indicating that FOLFOX is effective and has an acceptable toxicity profile as an adjuvant chemotherapeutic agent in patients with stage III and high-risk stage II colon cancer who have undergone a potentially curative resection.

\section{CONFLICT OF INTEREST}

No potential conflict of interest relevant to this article was reported.

\section{REFERENCES}

1. Jung KW, Park S, Kong HJ, Won YJ, Lee JY, Park EC, et al. Cancer statistics in Korea: incidence, mortality, survival, and prevalence in 2008. Cancer Res Treat 2011;43:1-11.

2. Obrand DI, Gordon PH. Incidence and patterns of recurrence following curative resection for colorectal carcinoma. Dis Colon Rectum 1997;40:15-24.

3. Moertel CG, Fleming TR, Macdonald JS, Haller DG, Laurie JA, Goodman PJ, et al. Levamisole and fluorouracil for adjuvant therapy of resected colon carcinoma. N Engl J Med 1990;322:352-8.

4. Wolmark N, Rockette H, Mamounas E, Jones J, Wieand S, Wickerham DL, et al. Clinical trial to assess the relative efficacy of fluorouracil and leucovorin, fluorouracil and levamisole, and fluorouracil, leucovorin, and levamisole in patients with Dukes' B and C carcinoma of the colon: results from National Surgical Adjuvant Breast and Bowel Project C-04. J Clin Oncol 1999;17:3553-9.

5. Andre T, Colin P, Louvet C, Gamelin E, Bouche O, Achille E, et al. Semimonthly versus monthly regimen of fluorouracil and leucovorin administered for 24 or 36 weeks as adjuvant therapy in stage II and III colon cancer: results of a randomized trial. J Clin Oncol 2003;21:2896-903.

6. de Gramont A, Figer A, Seymour M, Homerin M, Hmissi A, Cassidy J, et al. Leucovorin and fluorouracil with or without oxaliplatin as first-line treatment in advanced colorectal cancer. J Clin Oncol 2000;18:2938-47.
7. Goldberg RM, Sargent DJ, Morton RF, Fuchs CS, Ramanathan RK, Williamson SK, et al. A randomized controlled trial of fluorouracil plus leucovorin, irinotecan, and oxaliplatin combinations in patients with previously untreated metastatic colorectal cancer. J Clin Oncol 2004;22:23-30.

8. Andre T, Boni C, Mounedji-Boudiaf L, Navarro M, Tabernero J, Hickish T, et al. Oxaliplatin, fluorouracil, and leucovorin as adjuvant treatment for colon cancer. N Engl J Med 2004;350:2343-51.

9. Andre T, Boni C, Navarro M, Tabernero J, Hickish T, Topham C, et al. Improved overall survival with oxaliplatin, fluorouracil, and leucovorin as adjuvant treatment in stage II or III colon cancer in the MOSAIC trial. J Clin Oncol 2009;27:3109-16.

10. Sadahiro S, Suzuki T, Ishikawa K, Nakamura T, Tanaka Y, Masuda $\mathrm{T}$, et al. Recurrence patterns after curative resection of colorectal cancer in patients followed for a minimum of ten years. Hepatogastroenterology 2003;50:1362-6.

11. Sargent DJ, Wieand HS, Haller DG, Gray R, Benedetti JK, Buyse $M$, et al. Disease-free survival versus overall survival as a primary end point for adjuvant colon cancer studies: individual patient data from 20,898 patients on 18 randomized trials. J Clin Oncol 2005; 23:8664-70.

12. Sargent D, Shi Q, Yothers G, Van Cutsem E, Cassidy J, Saltz L, et al. Two or three year disease-free survival (DFS) as a primary endpoint in stage III adjuvant colon cancer trials with fluoropyrimidines with or without oxaliplatin or irinotecan: data from 12,676 patients from MOSAIC, X-ACT, PETACC-3, C-06, C-07 and C89803. Eur J Cancer 2011;47:990-6.

13. Kuebler JP, Wieand HS, O'Connell MJ, Smith RE, Colangelo LH, Yothers $\mathrm{G}$, et al. Oxaliplatin combined with weekly bolus fluorouracil and leucovorin as surgical adjuvant chemotherapy for stage II and III colon cancer: results from NSABP C-07. J Clin Oncol 2007;25:2198-204.

14. Biffi R, Orsi F, Grasso F, De Braud F, Cenciarelli S, Andreoni B. Catheter rupture and distal embolisation: a rare complication of central venous ports. J Vasc Access 2000;1:19-22.

15. Teichgraber UK, Pfitzmann R, Hofmann HA. Central venous port systems as an integral part of chemotherapy. Dtsch Arztebl Int 2011; 108:147-53. 